\title{
Superplastic Roll Forming of Axial Symmetric Articles from Superalloys
}

\author{
Farid Z. Utyashev" ${ }^{a}$ Shamil Kh. Mukhtarov ${ }^{b}$, Ayrat A. Nazarovc, \\ Radik R. Mulyukov ${ }^{d}$ \\ Institute for Metals Superplasticity Problems of the Russian Academy of Sciences, 39 Khalturin \\ street, Ufa 450001 Russia \\ afz1947@mail.ru, bshamil@anrb.ru, chazarov@imsp.da.ru, 'radik@anrb.ru
}

Key words: Superalloys, nanoparticles, superplasticity

\begin{abstract}
A new method of microstructure design in axisymmetric gas turbine engine components made of superalloys is proposed. The method is based on obtaining three types of microstructures by means of superplastic roll forming: coarse-grained one with coherent nano-sized precipitates of the $\gamma^{\prime}$ - phase, the second with ultrafine grains of both phases and the third combining coarse deformed matrix grains surrounded by thin layers of fine matrix grains and coarsened precipitates of $\gamma^{\prime}$ - phase.
\end{abstract}

\section{Introduction}

According to the modern classification of materials with respect to the sizes of their structural constituents, the heat resistant nickel alloys used in the construction of aircraft engines can be regarded as essentially nanostructured structural materials. Indeed, nanostructured materials are those materials in which the sizes of structural constituents (grains, strengthening phases, composite inclusions, coatings etc.) are of the order or less than $100 \mathrm{~nm}$. In superalloys, these elements consist of nanoparticles of intermetallic $\gamma^{\prime}$ - phase, which play the major strengthening role. The size and morphology of the intermetallic particles essentially determine the technological and mechanical properties of the superalloys and to control these characteristics is very important in the processing and exploitation of engine parts out of these alloys. In this paper, on an example of a superalloy EP 962 (Russian analogue of Rene 95) we show the possibility of controlling the size and morphology of intermetallic particles by thermal treatment and plastic deformation with a related variation of the mechanical properties in the range from a superplastic behavior in the stage of fabrication of articles to controllable properties required by the exploitation conditions.

\section{Problem formulation}

In the use of superalloys there is always a dual problem related to the necessities to enhance their exploitation and technological properties. The former should be increased to a level that meets the requirements which are imposed to the aircraft engines of new generation, while the latter mean the increase of plasticity and reduction of strain resistance. Low plasticity results in an increase of the labor intensity of fabrication and the price of discs while low exploitation properties result in lowering of their quality and reliability. One of the ways to solve this problem is the control of regimes of treatment and the structural state of the alloys in the process of fabrication of standard components of gas turbine engines (GTEs) [1].

\section{Problem solution}

Normally, the problem of optimization of the structure in alloys is solved in relation to the exploitation properties. In this regard, only some general requirements to the structure are made: it must provide the guaranteed operational life of the articles in given exploitation conditions. Structural parameters necessary for this are obtained experimentally by fitting the chemical, 
granular and phase structure, testing mechanical properties on samples and full-scale testing of the components. The optimization of the structure and properties of disc alloys is associated with a big volume of expensive experiments. In this work we had a particular goal to work out a method of obtaining a specified structure in discs that would provide its different parts subjected to essentially different exploitation requirements adequately different properties and the possibility to optimize the structure in different disc alloys on the basis of this method.

With this goal, a heavy-alloyed heat resistant alloy EP 962 has been chosen in which three structural states have been aimed to obtain during the processing of a $550 \mathrm{~mm}$-diameter disc. The first state is a coarse-grained structure with coherent precipitates of the strengthening $\gamma^{\prime}-$ phase. The second state is a structure consisting of ultrafine matrix grains $\left(d_{\gamma} \approx 2-3 \mu \mathrm{m}\right)$, comparable-sized precipitates of the most volume of the $\gamma^{\prime}$ - phase and disperse precipitates of the rest part of the $\gamma^{\prime}$-phase. Such a structure is usually called a microduplex one. At high temperatures corresponding to the stationary regimes of the exploitation of the alloy, the coarse-grained state provides the alloy the highest heat resistance, while the ultrafine-grained (UFG) state gives it superplastic properties, that is, lower strain resistance and higher strains. At lower temperatures, for example, at room ones, the both states provide the alloys a high strain resistance, but in the UFG state the strength of the alloy is considerably higher than in the coarse-grained state.

As the third state we have chosen a "necklace" structure which combines coarse deformed matrix grains surrounded by thin layers of fine matrix grains and coarsened precipitates of $\gamma^{\prime}$ - phase. As known, such a structure provides the superalloys an optimal combination of long- and short-term properties at sufficiently high and ambient temperatures.

Roll-forming has been chosen as a method of disc processing with the specified structures (Fig. 1). This process allows deforming a blank in a wide interval of temperatures, strain rates and strains, automated maintaining and changing of the processing conditions (temperature and strain rate). In accordance with the task, in the disc rim which undergoes high-temperature exploitation conditions a coarse grained structure was formed, in the disc web that is less heated during the exploitation a necklace structure and in the even less heated but more stressed disc hub the fine grained structure were formed.

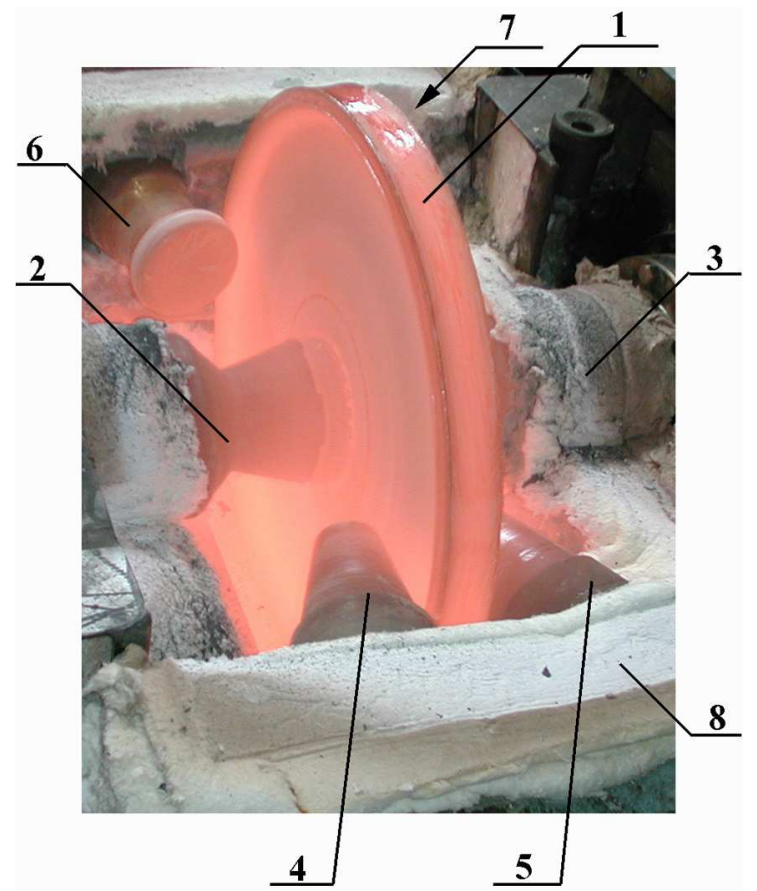

Fig. 1. Rolling zone in the mill SRD 800: 1 - disc under rolling; 2, 3 - tail spindles rotating the disc; 4-7 - deforming tail spindles rotating around their axis and moving along the rolling plane; 8 - the bottom part of the furnace in which superplastic deformation is carried out.

The upper part of the furnace is removed to take a photo. 
Formation of a specified structure. From the theory and practice of treatment of heat resistant alloys it is known that refinement of the matrix grains and transformation of phase boundaries in superalloys is possible only by deformation, while the backward process of transformation of the UFG structure to a coarse-grained one can be done by thermal treatment. Since in the as-received condition the deformable cast superalloys including EP 962 have the coarse-grained structure, the task of the first treatment is to form a UFG structure in the alloy. Basically, this task is reduced to the transformation of coherent $\gamma / \gamma^{\prime}$ interphase boundaries to non-coherent ones having high misorientation angles. Such a transformation cannot be realized by thermal treatment. In particular, long holding of the alloy in $\gamma$ - phase field and / or slow cooling from $\gamma$ to $\left(\gamma+\gamma^{\prime}\right)$ - phase field result only in a coagulation of particles and a partial violation of the coherency of $\gamma / \gamma^{\prime}$ interphase boundaries (Fig. 1).

An increase of the misorientation angles of these boundaries occurs during dynamic and / or primary recrystallization leading to the growth of particles precipitated on low-mobility boundaries of recrystallized matrix grains. Basically, in superalloys, like in other materials, an in-situ recrystallization is possible that occurs without the boundary migration and results in the formation of nanograins in the matrix. This occurs, for instance, during high pressure torsion. However, this deformation process is technologically non-efficient and with respect to bulk billets is practically unfeasible. To form a UFG structure in large billets a process has been developed that consists of multi-step upsetting with a stepwise temperature reduction [2]. Using this method, pancake-shaped forged blanks with the diameter $410 \mathrm{~mm}$ having a microduplex structure have been obtained (Fig. 2) from which the GTE discs further were roll formed.
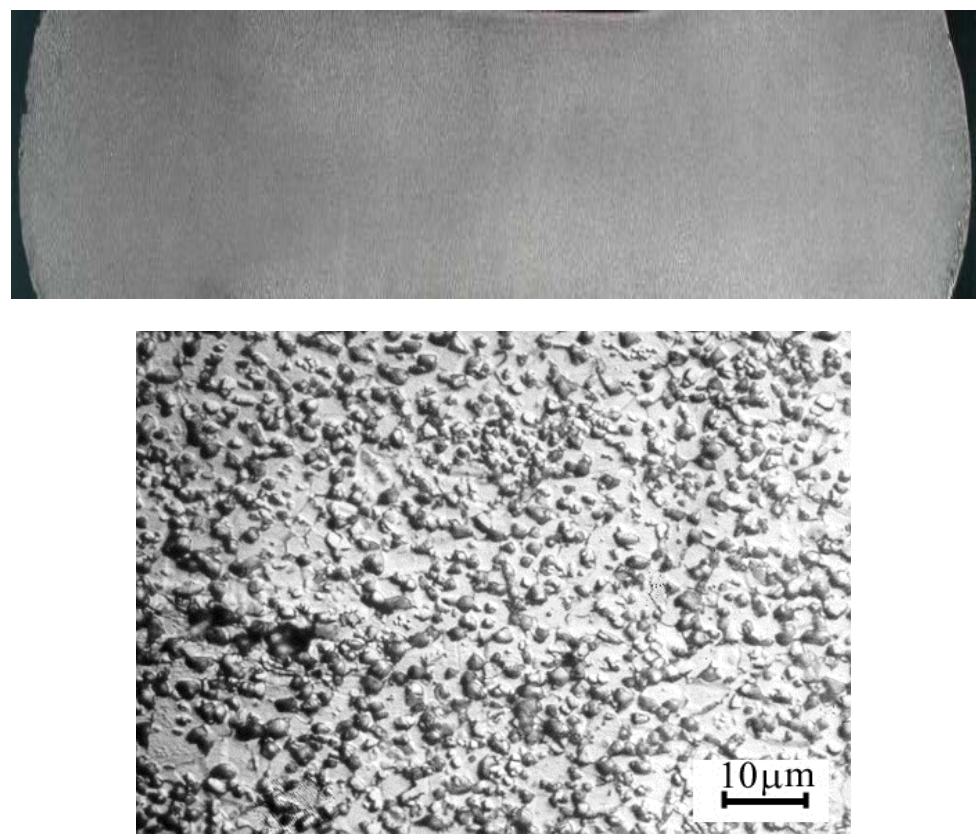

Fig. 2. Typical microduplex-type ultrafine-grained structure in superalloys. At the topmacroscopic view (4-fold reduced photo), beneath - the microstructure.

The roll forming process was carried out on the roll-forming mill SRD 800. Taking the advantage of this process, the deformation was done in a gradient temperature field that was realized by an air blow through tail spindles and central hole in the hub of the disc workpiece. In the disc hub a relatively low temperature favoring the stable microduplex structure was maintained, the web was heated to a higher temperature that provided a moderate grain growth and the deformation in the two-phase field and last, the roll forming of the rim was done at the highest temperature close to the temperature of the full dissolution of the $\gamma^{\prime}$ - phase that provided the most intense matrix grain growth. 
As a result of roll forming and following thermal treatment using incomplete quenching a disc was obtained, which had a fine-grained structure with $d_{a v} \approx 30 \mu \mathrm{m}$ in the hub, a mixed necklace structure in the web and a coarse-grained structure with wavy grain boundaries in the rim.

Mechanical properties of the disc with a specified structure. Mechanical properties of the disc in its different parts are presented in Fig. 3a, b. One can see that the coarse-grained structure in the rim and the necklace structure in the web possess fairly high heat resistance properties, while the fine grained structure in the hub is characterized by a high strength. Such a distribution of the properties corresponds to the distribution of the stresses in fast-rotating discs of equal cross-section in which the maximum load is experienced by the hub part. It should be also noted that the longterm properties of the alloy in the hub part are still high enough even in the case of heating this part up to temperatures about $650^{\circ} \mathrm{C}$ that are uncharacteristic for the exploitation of this component, while at lower temperatures, say, $500^{\circ} \mathrm{C}$, the service life of the microduplex alloy, as has been shown earlier, dozens times exceeds the required characteristics [1].

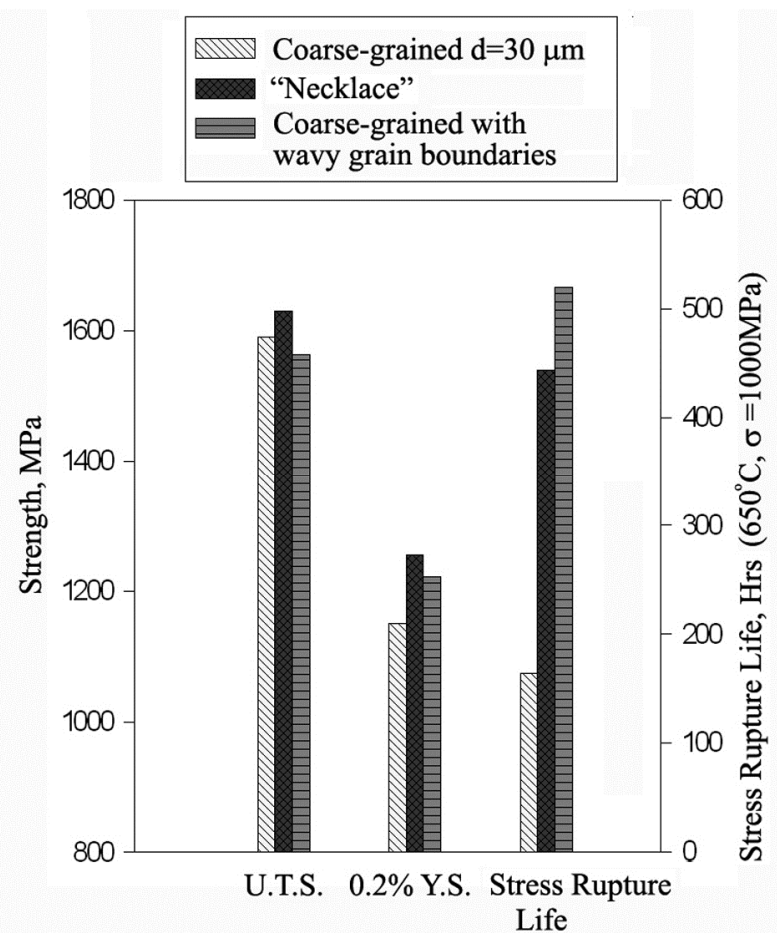

(a)

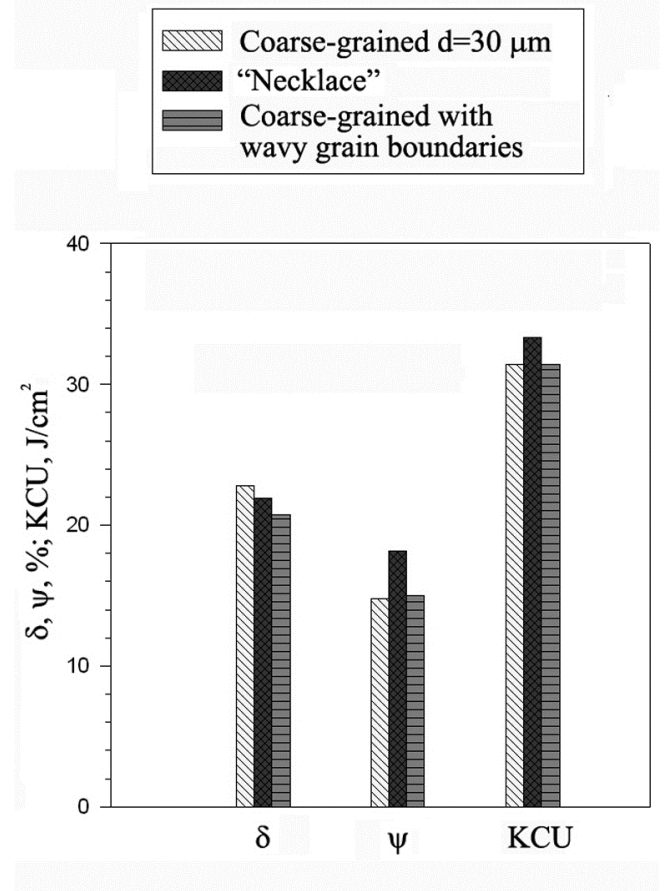

(b)

Fig. 3. Strength and heat resistance properties of the EP 962 alloy with different types of microstructure.

\section{Summary}

The present work shows that roll forming in the conditions of superplasticity allows one to obtain, during the process of forming, specified structural states in discs made of superalloys. The formation of specified variable structure is an essentially new method of structural design of discs that is capable of giving them a uniform strength not only by a macroscopic design of the shape and dimensions, but also by a mesoscopic structure control specifying the sizes and morphologies of structural elements. This method can be used to decrease the weight of parts. Moreover, in a combination with a rational thermal treatment it is a promising method of the formation of optimal structure and set of mechanical properties in the discs. 
Along with the above mentioned possibilities the superplastic roll-forming allows one to process discs also in the traditional way, with uniform structure and properties over the whole component. In this case the method has the following advantages:

- roll forming of figurine-shaped articles;

- minimum consumption of metal, energy and labor, the metal recovery higher that $50 \%$ and deformation load in the range 10 to 50 tons;

- automated processing of a wide range of axisymmetric parts of GTE with the diameter up to $800 \mathrm{~mm}$ by means of a universal tool and without an equipment realignment and use of big dies. For roll forming a standardized forged blank is used:

- environmental safety: no noise, vibrations, harmful gas pollution, heat radiation and other negative effects.

\section{References}

[1] O.A. Kaibyshev and F.Z. Utyashev: Superplasticity: Microstructural Refinement and Superplastic Roll Forming (Futurepast, Arlington, Virginia, USA 2005).

[2] F.Z. Utyashev, O.A. Kaibyshev and V.A. Valitov, Method of fabrication of axisymmetric parts and method of processing blanks of multiphase alloys (variants). Russian patent \#2119842. 